ARTIGO ORIGINAL

\title{
HANSENÍASE NA REGIÃO NORTE DO BRASIL: PERFIL CLÍNICO EPIDEMIOLÓGICO ENTRE 2015 E 2017
}

\section{HANSENIASIS IN THE NORTHERN BRAZIL REGION: EPIDEMIOLOGICAL CLINICAL PROFILE BETWEEN 2015 AND 2017}

Rita de Cássia do Amor Divino Ramalho Gama; Barbara Barros Andrade²; Barbara Alves de Brito²; Juliane Laura Tonzar Sanches²; Letícia Roxadelli Inácio²; Rafaela de Sá Gondolo²; Vitória Gewehr Fucuta²; Kássia Dorneles Silva ${ }^{3}$

\begin{abstract}
RESUMO
Objetivo: analisar o perfil epidemiológico clínico da hanseníase na região norte do Brasil entre os anos de 2015 a 2017. Método: Trata-se de um estudo epidemiológico, descritivo, transversal e retrospectivo, onde buscou-se por meio de dados disponíveis no Departamento de Informática do Sistema Único de Saúde (DATASUS/TABNET), aqueles referentes ao número de nervos afetados no diagnóstico, grau de progressão da doença, forma clínica e número de lesões cutâneas da hanseníase na região Norte do Brasil, no período de 2015 a 2017. Resultados: O perfil epidemiológico clínico da hanseníase na região Norte do Brasil, entre os anos de 2015 a 2017, foi estruturado a partir dos 3.340 casos notificados ao Sistema Nacional de Agravos de Notificação, onde pode-se observar que o ano de 2016 foi o que ocorreu maior número de notificações, sendo a forma dimorfa a mais relatada em todos os anos. Na maior parcela das notificações, foi determinado que não havia nenhum nervo acometido, com um grau zero de progressão e com duas a cinco lesões cutâneas. Conclusão: O diagnóstico na fase dimorfa da hanseníase revela que há negligência nas estratégias de rastreio da doença na região, haja vista que, esta forma clínica corresponde a estágios mais avançados da doença, com maior potencial gerador de incapacidades. $O$ fato de haver um paradoxo entre a forma clínica mais prevalente, dimorfa, e o número de nervos afetados, zero, permite inferir que seja um reflexo da notificação com dados insuficientes da doença, ou mesmo subnotificações. Este fato merece atenção na saúde pública, uma vez que inviabiliza o conhecimento da real situação da doença na região, dificultando a estruturação de políticas públicas que atendam as demandas por promoção e prevenção em saúde, principalmente em relação aos contatos dos portadores, representando o surgimento de novos casos e prorrogação do ciclo de transmissão
\end{abstract}

Palavras-chave: Hanseníase; Epidemiologia; Prevenção Primária

\section{ABSTRACT}

Objective: To analyze the clinical epidemiological profile of leprosy in the northern region of Brazil between 2015 and 2017. Method: This is a descriptive, cross-sectional and retrospective epidemiological study using data available from the Department of Informatics of the Unified Health System (DATASUS / TABNET), those referring to the number of nerves affected in the diagnosis, degree of disease progression, clinical form and number of leprosy skin lesions in the northern region of Brazil, from 2015 to 2017. Results: The clinical epidemiological profile of leprosy in the northern region of Brazil, from 2015 to 2017, was structured from the 3,340 cases notified to the National System of Notification Disorders, where it can be observed that 2016 was the year. the highest number of notifications occurred, being the most reported dimorphic form in all years. In most of the reports, it was determined that there was no nerve affected, with a zero degree of progression and with two to five skin lesions. Conclusion: The diagnosis in the dimorphic phase of leprosy reveals that there is neglect in the screening strategies of the disease in the region, since this clinical form corresponds to more advanced stages of the disease, with greater potential for disability. The fact that there is a paradox between the most prevalent clinical form, dimorphic, and the number of affected nerves, zero, allows us to infer that this is a reflection of the underreporting of the disease. This fact deserves attention in public health, since it makes unfeasible the knowledge of the real situation of the disease in the region, making it difficult to structure public policies that meet the demands for health promotion and prevention, especially regarding the contacts of carriers, representing the emergence new cases and extension of the transmission cycle.

Keywords: Hanseníase; Epidemiologia; Prevenção Primária 
INTRODUÇÃO

A hanseníase é uma doença infectocontagiosa, com alta infectividade. A clínica desta doença é variável de acordo com a resposta do hospedeiro frente ao agente etiológico da doença, o bacilo Mycobacteruim Leprae. Desta forma, a fim de uma melhor caracterização clínica da doença, de modo a permitir uma melhor definição do plano terapêutico a ser instituído, tem-se estabelecido diversas formas de caracterização da doença, considerando o número de lesões encontradas no paciente, a forma destas lesões, o número de nervos acometidos, dentre outros ${ }^{1}$.

Uma das principais características da hanseníase é a evolução insidiosa, com predileção para os nervos periféricos assim como células da pele. A localização do início das lesões é variável, apresentando uma evolução na percepção nervosa sensitiva do local, sendo inicialmente a sensibilidade térmica, evoluindo para a deleção da sensibilidade nociceptiva, depois a tátil, ponto em que a doença já está em estágio avançado e o paciente pode desenvolver sinais como a parestesia e plegias musculares na região acometida ${ }^{2}$.

Uma vez que a evolução da doença é lenta e progressiva, percebe-se uma grande dificuldade no diagnóstico precoce, assim como no início do tratamento. A perda da sensibilidade torna estes pacientes não diagnosticados mais susceptíveis a acidentes e consequentemente a lesões como queimaduras ou outros ferimentos. Outro fato preocupante em relação a essa dificuldade diagnóstica é a maior probabilidade de disseminação da doença para outros contactantes, uma vez que o bacilo causador da hanseníase apresenta alto poder de contágio ${ }^{3}$.

\section{OBJETIVOS}

Desta forma, este estudo tem como objetivo analisar o perfil epidemiológico clínico da hanseníase na região norte do Brasil entre os anos de 2015 a 2017, assim como, determinar a quantidade de nervos afetados durante os diagnósticos feitos na região, qual o grau de progressão da doença durante a avaliação diagnóstica, a forma clínica e o número de lesões cutâneas nestes pacientes.

\section{MÉTODO}

Trata-se de um estudo epidemiológico, descritivo, transversal e retrospectivo, onde buscou-se por meio de dados disponíveis no Departamento de Informática do Sistema Único de Saúde (DATASUS/TABNET), aqueles referentes ao número de nervos afetados no diagnóstico, grau de progressão da doença, forma clínica e número de lesões cutâneas da hanseníase na região Norte do Brasil, no período de 2015 a 2017. Os dados foram tabelados em Excel e comparados por meio de avaliações estatísticas de frequência absoluta e incidente, a fim de determinar um padrão clínico da doença na região durante o período analisado.

\section{RESULTADOS}

Durante o período analisado, 3.340 casos de hanseníase foram notificados ao DATASUS na região Norte do País, sendo o ano de 2016 com o maior número de diagnósticos, com 40,2\% dos casos ( $n=1.342)$. O tipo de forma clínica mais incidente foi a dimorfa em todos os anos. 0 gráfico 1 apresenta a incidência das formas clínicas entre os anos. Observa-se também que a segunda forma mais incidente, a indeterminada, apresentou uma redução de $1,5 \%$ na incidência durante o período analisado $(n=52)$.

Gráfico 1: Incidência das formas clínicas de casos de hanseníase notificados entre 2015 e 2017, Tocantins, Brasil.

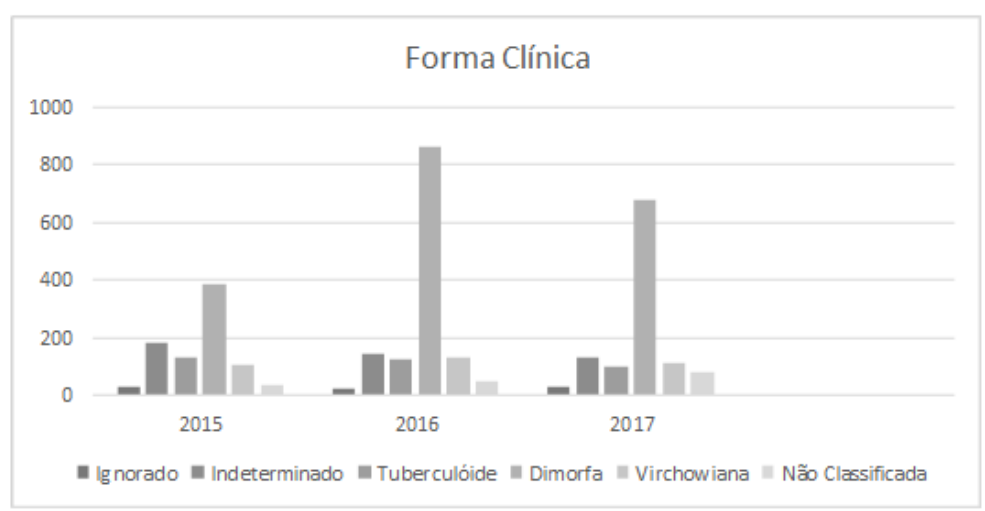

Em relação ao número de nervos afetados, apenas $53,6 \%$ dos casos notificados relataram esta variável a qual, foi dividida em três grupos: nenhum, até cinco e mais que cinco. Dentre estas três categorias, a maior incidência foi do grupo zero durante os três anos, com $69 \%$ dos casos onde o número de nervos acometidos foi notificado. Foi observado um crescimento nos outros dois grupos durante os anos, como demonstrado no gráfico 2 .

Gráfico 2: Número ( $n$ ) de nervos acometidos de pacientes hansênicos notificados entre 2015 e 2017, Tocantins, Brasil.

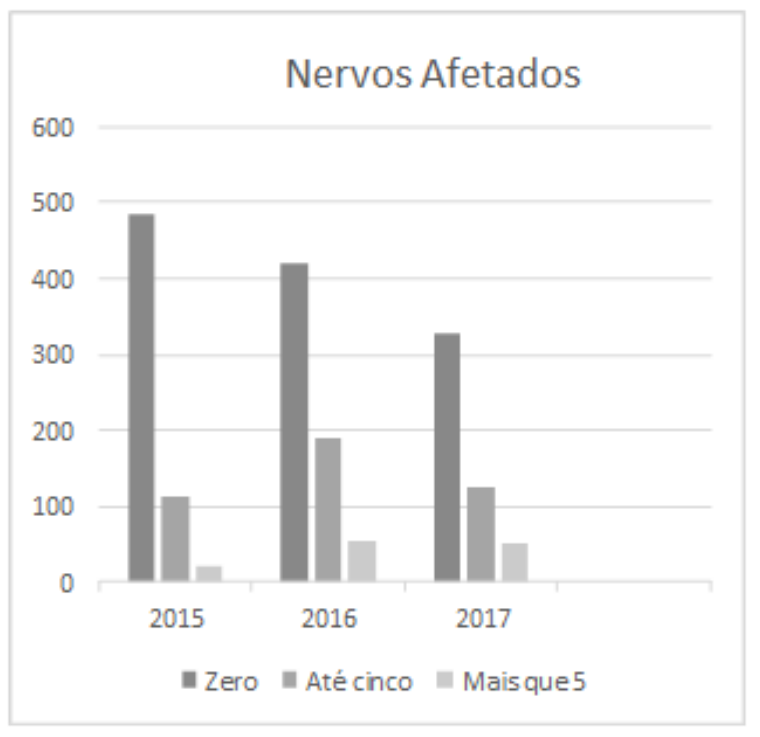

O grau de progressão da doença mais incidente na avaliação diagnóstica foi o grau zero, com aproximadamente $53 \%$ do total, enquanto que o grau II apresentou a menor incidência, com 7\%. Cabe destacar que em 7,5\% $(n=250)$ dos casos, o grau de progressão da doença não foi avaliado ou notificado. Em relação ao número de lesões cutâneas, foram 
agrupadas em: nenhuma lesão; lesão única; 2 a 5 lesões; mais que cinco lesões e; branco ou ignorado. Dentre estes grupos, a maior incidência foi de pacientes com 2 a 5 lesões, com $37 \%$ dos casos ( $n=1230)$. O gráfico 3 apresenta os dados em relação ao número de lesões na avaliação diagnóstica da doença.

Gráfico 3: Número (n) de lesões cutâneas no diagnóstico inicial de casos hansenicos notificados entre 2015 e 2017, Tocantins, Brasil.

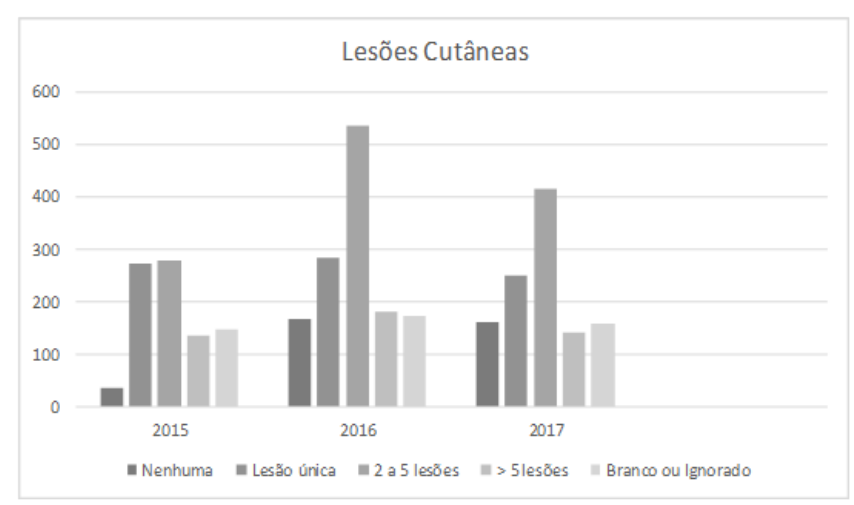

\section{DISCUSSÃO}

A forma dimorfa da hanseníase é caracterizada por múltiplas lesões na pele, na forma de manchas, de coloração avermelhada ou esbranquiçada, com bordas elevadas. O acometimento dos nervos periféricos já se encontra avançado, podendo ocorrer de forma assimétrica, havendo perda total ou parcial da sensibilidade e disfunção autonômica ${ }^{4}$.

Os dados analisados permitiram evidenciar que a forma dimorfa foi a mais frequente na região norte durante o período estudado, o que vai de acordo com outros estudos ${ }^{5-7}$. Uma vez que esta forma clínica da doença é decorrente de um longo período de incubação, em torno de dez anos ou mais ${ }^{4}$, é possível inferir que a falha no rastreio da hanseníase na região Norte do Brasil se apresenta como um fator de mau prognóstico para os pacientes da região, uma vez que, o acometimento neural avançado, aumenta a probabilidade de acidentes e possíveis sequelas/incapacidade advindas da doença ${ }^{8}$.

Cabe destacar que o diagnóstico precoce faz parte das estratégias propostas pelo Ministério da Saúde, que rege o Sistema Único de Saúde (SUS), norteando ações de promoção e prevenção em saúde. Entretanto, o número de nervos periféricos acometidos encontrados nesta pesquisa, resultou em um paradoxo em relação à apresentação clínica e contraria, em princípio, o entendimento de que a forma dimorfa, mais prevalente nos dados, tem como característica um grande acometimento neural e ainda a presença de múltiplas lesões, entretanto, nos dados encontrados, o número de nervos acometidos mais prevalente foi zero e o número de lesões mais prevalentes também foi zero.

Assim, vale ponderar duas situações relevantes: é elevado o percentual $(46,4 \%)$ de notificações no Datasus sem a informação acerca do nervo afetado; e dentre os casos onde a notificação da quantidade de nervos acometidos ocorreu, $69 \%$ foi notificado que não havia nervo acometido.

Um viés para o entendimento deste paradoxo, parte do fato que $53,6 \%$ das notificações estavam incompletas no tocante ao número de nervos acometidos e que, no decorrer dos anos, os grupos de 3 a 5 nervos e mais que 5 nervos acometidos apresentou aumento na frequência, o que indica uma melhora na qualidade das notificações. Esta, hipoteticamente, poderia ser uma das razões. Porém, destaca-se no presente estudo as implicações destas possíveis falhas quando da coleta de informações que geram as notificações, podendo gerar graves prejuízos no controle epidemiológico desta doença negligenciada e ainda muito presente na região Norte do País. De acordo com Cardoso e Clemente ${ }^{9}$ (p.33), "as informações não registradas, podem se caracterizar como subnotificações, ou seja, dados não registrados pelo serviço local de saúde ou não foi notificado no tempo estabelecido". Para os autores, a subnotificação pode ser reflexo de uma gama de atribuições, podendo destacar: (1) diminuto conhecimento técnicocientífico por parte do profissional responsável por tratar as informações, no que é inerente à informações conceituais da epidemiologia; (2) a falta de comunicação entre o profissional que realizou o diagnóstico e o profissional responsável por alimentar o sistema com as informações colhidas e; (3) pedido de sigilo de diagnóstico ${ }^{9}$.

Desta forma, a presença de dados subnotificados no Sistema Nacional de Agravos de Notificação (SINAN), culmina em um forte problema de saúde pública, tendo em vista que, acaba por impedir um reconhecimento fidedigno das condições sanitárias e epidemiológicas da região por parte dos gestores de políticas públicas, dificultando a estruturação e embasamento de políticas públicas eficazes frente aos problemas enfrentados pela população em questão, deixando de subsidiar medidas de maior afinco na prevenção e promoção em saúde, pilares da básicos da atenção primária à saúde e essencial no projeto do SUS ${ }^{10-11}$.

Ao considerar o fato de a hanseníase apresentar alto grau infectante assim como uma dificuldade no diagnóstico precoce, devido ao curso lento e insidioso, preconiza-se que as ações para a promoção e prevenção em saúde estejam voltadas principalmente para o controle de indivíduos em contato com os infectados, minimizando os riscos de contágio e propagação da doença ${ }^{12-13}$.

Na pesquisa de Leano e colaboradores ${ }^{14}$, objetivou-se analisar a tendência dos indicadores epidemiológicos da hanseníase no que se relaciona com o prognóstico e desenvolvimento de sequelas e incapacidades físicas. Os autores encontraram uma alta incidência de pacientes com idades menores que 15 anos, dando relevância a este dado, demonstrando que, este fato pode ser reflexo da negligência diagnóstica dos casos, aumentando o número de contactantes com doentes, seja no ambiente domiciliar ou extra domiciliar ${ }^{15-16}$. 


\section{CONCLUSÃO}

O PERFIL EPIDEMIOLÓGICO CLÍNICO DA HANSENÍASE NA REGIÃO NORTE DO BRASIL, ENTRE OS ANOS DE 2015 A 2017, FOI ESTRUTURADO A PARTIR DOS 3.340 CASOS NOTIFICADOS AO SISTEMA NACIONAL DE AGRAVOS DE NOTIFICAÇÃO, ONDE PODE-SE OBSERVAR QUE O ANO DE 2016 FOI O QUE OCORREU MAIOR NÚMERO DE NOTIFICAÇÕES, SENDO A FORMA DIMORFA A MAIS RELATADA EM TODOS OS ANOS. NA MAIOR PARCELA DAS NOTIFICAÇÕES, FOI DETERMINADO QUE NÃO HAVIA NENHUM NERVO ACOMETIDO, COM UM GRAU ZERO DE PROGRESSÃO E COM DUAS A CINCO LESÕES CUTÂNEAS.

A PESQUISA PERMITIU ELUCIDAR UMA FALHA, DE GRANDE RELEVÂNCIA PARA A SAÚDE PÚBLICA DA REGIÃO E DO BRASIL, EM RELAÇÃO ÀS NOTIFICAÇÕES REALIZADAS, UMA VEZ QUE, A FORMA DIAGNÓSTICA MAIS PREVALENTE, DIMORFA, APRESENTA QUADRO CLÍNICO QUE DESTOA DOS DEMAIS DADOS, PRINCIPALMENTE EM RELAÇÃO AO NÚMERO DE NERVOS AFETADOS. ESTE FATO PODE ESTAR, HIPOTETICAMENTE, VINCULADO AO FATO DE AS NOTIFICAÇÕES NÃO TEREM SIDO PREENCHIDAS COMPLETAMENTE, O QUE FOI EVIDENCIADO PELO ALTO TEOR DE CAMPOS EM BRANCO NAS NOTIFICAÇÕES AO SINAN.

ADEMAIS, PERCEBE-SE QUE ESTE QUADRO NÃO É ÚNICO DA REGIÃO NORTE, ESTANDO PRESENTE EM DIVERSOS OUTROS ESTUDOS. O FATO DE NÃO HAVER UM SISTEMA CONFIÁVEL DE NOTIFICAÇÃO DA DOENÇA, ACARRETA PREJUÍZOS PRINCIPALMENTE A POPULAÇÃO, UMA VEZ, INVIABILIZA A ESTRUTURAÇÃO DE POLÍTICAS PÚBLICAS DE SAÚDE QUE SEJAM EFETIVAS PARA A PROMOÇÃO E PREVENÇÃO DA HANSENÍASE, PRINCIPALMENTE AO SE PENSAR NOS CONTACTANTES, SENDO ESTES INDIVÍDUOS SUBMETIDOS A UM MAIOR GRAU DE RISCO EM SER ACOMETIDO PELA DOENÇA DEVIDO Á FALTA DE INFORMAÇÃO EM SAÚDE DA POPULAÇÃO, ASSIM COMO, DE POLÍTICAS QUE SUBSIDIEM PRÁTICAS EM AUTOCUIDADO PARA O MESMO.

O FATO DE HAVER UM PARADOXO ENTRE A FORMA CLÍNICA MAIS PREVALENTE, DIMORFA, E O NÚMERO DE NERVOS AFETADOS, ZERO, PERMITE INFERIR QUE SEJA UM REFLEXO DA SUBNOTIFICAÇÃO DA DOENÇA. ESTE FATO MERECE ATENÇÃO NA SAÚDE PÚBLICA, UMA VEZ QUE INVIABILIZA O CONHECIMENTO DA REAL SITUAÇÃO DA DOENÇA NA REGIÃO, DIFICULTANDO A ESTRUTURAÇÃO DE POLÍTICAS PÚBLICAS QUE ATENDAM AS DEMANDAS POR PROMOÇÃO E PREVENÇÃO EM SAÚDE, PRINCIPALMENTE EM RELAÇÃO AOS CONTATOS DOS PORTADORES, REPRESENTANDO O SURGIMENTO DE NOVOS CASOS E PRORROGAÇÃO DO CICLO DE TRANSMISSÃO.

\section{REFERÊNCIAS}

1. Alves CJ, Barreto JA, Fogagnolo L, et al. Avaliação do grau de incapacidade dos pacientes com diagnóstico de hanseníase em serviço de dermatologia do estado de São Paulo. Rev Soc Bras Med Trop Dis. 2010;43(4):460-1. doi:10.1590/S0037-86822010000400025

2. Oliveira DT. Avaliação de sequelas neurológicas em pacientes com hanseníase no estado de Sergipe [Dissertação]. Sergipe: Universidade Federal de Sergipe; 2012.
3, Rodrigues LC, Lockwood DN. Leprosy now: epidemiology, progress, challenges, and research gaps. Lancet Infect Dis. 2011 Jun;11(6):464-70. doi: 10.1016/S1473-3099(11)700068.

4. Brasil. Ministério da Saúde. Secretaria de Vigilância em Saúde. Departamento de Vigilância das Doenças Transmissíveis. Guia prático sobre a hanseníase/Ministério da Saúde, Secretaria de Vigilância em Saúde, Departamento de Vigilância das Doenças Transmissíveis. - Brasília: Ministério da Saúde, 2017. 68 p.

5. Melo JP, Moraes MM, Santos NR, et al. Perfil epidemiológico os casos de hanseníse de uma unidade de saúde. Rev Saude Colet UEFS. 2017;7(1):29-34.

6. Silva MS, Silva EP, Monteiro FF, et al. Perfil clínicoepidemiológico da hanseníase no estado do Acre: estudo retrospectivo. Hansen Int. 2014;39(2):19-26.

7. Araújo KMFA, Lana FCF, Paz LFA, et al. Hanseníase: a visibilidade da doença no idoso. In: Anais Congresso Internacional de Envelhecimento Humano (CIEH); 2015;2(1):1-7.

8. Chaves AEP, Araújo KMF, Nunes MLA, et al. Hanseníase em idosos no Brasil no ano de 2012. In: Congresso Internacional de Envelhecimento Humano (CIEH); Campina Grande. 2013.

9. Cardoso TV, Clemente CMS. Análise espacial dos casos de hanseníase no semiárido brasileiro (2010/2014). Rev Unimontes Cient. 2017;19(2):27-39 p.

10. Pinheiro RS, Andrade VL, Oliveira GP. Subnotificação da tuberculose no Sistema de Informação de Agravos de Notificação (SINAN): abandono primário de baculíferos e captação de casos em outras fontes de informação usando linkage probabilístico. Caderno Saúde Pública.2012;28(8):1559-1568 p.

11. Miranda CW, Chiaravalloti Neto F, Barrozo LV. Socioeconomic and environmental effects influencing the development of leprosy in Bahia, northeastern Brazil. Trop Med Int Health. 2014;19(12):1504-14 p.

12. Galvão PRS, Ferreira AT, Maciel MGG, et al. Uma Avaliação do sistema de informação SINAN usado no Programa de Controle de Hanseníase no estado do Pernambuco, Brasil. Cad Saúde Coletiva. 2009; 17(1):87102.

13. Penna MLF, Gross MAF, Rocha MCN, et al. Comportamento epidemiológico da hanseníase no Brasil. IN: Brasil. Ministério da Saúde. Secretaria de Vigilância em Saúde. Departamento de Análise de Situação de Saúde. Saúde Brasil 2009: uma análise da situação de saúde e da agenda nacional e internacional de prioridades em saúde. Ministério da Saúde, Brasília, 2010.

14. Leano HAM, Araújo KMFA, Rodrigues RN, et al. Indicadores relacionados a incapacidade física e diagnóstico de hanseníase. Rev Rene. 2017;18(6):832-839. 
15. Freitas BHBM, Cortela DCB, Ferreira SMB. Trend of leprosy in individuals under the age of 15 in Mato Grosso (Brazil), 2001-2013. Rev Saúde Pública. 2017;51(2).

16. Santos SD, Penna GO, Costa MCN, et al. Leprosy in children and adolescents under 15 years old in an urban centre in Brazil. Mem Inst Oswaldo Cruz. 2016; 111(6):35964. 